Results Key concepts and processes were synthesised into a framework of change processes in group-based health interventions. Processes were categorised into: (1) group dynamic and development processes, (2) inter-personal change processes, and (3) intra-personal change processes in groups. Examples of practical strategies to facilitate and manage these processes were identified and categorised into: (1) group design elements, (2) group set-up tasks, (3) group facilitation strategies, and (4) group closure tasks. Work to identify examples of how group interaction and group processes might link with participant engagement and outcomes is still in progress but will be presented.

Discussion The presented framework integrates a large body of literature on change processes in groups, and provides examples of practical strategies that can be used to instigate and facilitate change processes in group-based health interventions. It provides a practical tool for researchers and practitioners that can be used to design and deliver better groupbased health interventions, train group facilitators, and guide evaluations of group-based interventions, with a view to optimising intervention engagement and outcomes.

\section{P92 LIFESTYLE INTERVENTIONS FOR THE TREATMENT OF OVERWEIGHT/OBESE ADOLESCENTS-COCHRANE REVIEW}

${ }^{1}$ LA-K Al Khudairy*, ${ }^{2}$ EL Loveman, ${ }^{2} \mathrm{JC}$ Colquitt, ${ }^{3} \mathrm{EM}$ Mead, ${ }^{1} \mathrm{RJ}$ Johnson, ${ }^{1} \mathrm{HR}$ Fraser, ${ }^{3}$ OJ Joan Olajide, ${ }^{1} \mathrm{MM}$ Murphy, ${ }^{1} \mathrm{RV}$ Velho, ${ }^{4} \mathrm{CO}$ O'Malley, ${ }^{3} \mathrm{LA}$ Azevedo, ${ }^{3} \mathrm{LE}$ Ells, ${ }^{5}$ MIM Metzendorf, ${ }^{1} \mathrm{KR}$ Rees. 'Division of Health Sciences, Warwick Medical School, Coventry, UK; ${ }^{2}$ Effective Evidence LLP, Waterlooville, UK; ${ }^{3}$ Health and Social Care Institute, Teeside University, Middlesbrough, UK; ${ }^{4}$ School of Medicine, Pharmacy and Health, Durham University Queen's Campus, Stockton-on-Tees, UK; ${ }^{5}$ Cochrane Metabolic and Endocrine Disorders Group, Medical Faculty of Heinrich-heine-University Dusseldorf, Dusseldorf, Germany

\subsection{6/jech-2017-SSMAbstracts. 193}

Background The prevalence of overweight and obese adolescents has increased worldwide, presenting a global public health crisis. This review assessed the efficacy of diet, exercise and behavioural interventions for the treatment of overweight/ obesity in adolescents (12-17 years).

Methods A systematic literature search (up to July 2016) with no language restrictions was performed in CENTRAL, MEDLINE, EMBASE, PsycINFO, CINAHL, LILACS, and the trial registers ClinicalTrials.gov and ICTRP Search Portal. Search terms included obesity, diet, exercise and adolescent. References of identified studies and systematic reviews were checked. Authors of included studies were contacted for missed studies. Two reviewers evaluated studies independently at all stages. Eligibility: RCTs that observed participants for $\geq$ six months, overweight/obese (investigator-assessed) adolescents (mean age 12-17 years), interventions with a primary aim to treat overweight/obesity with any form of dietary, exercise and/or behavioural therapy delivered as a single or multi component intervention, any setting and any delivery method. Comparators were no treatment/wait list control, usual care or an alternative concomitant therapy providing it is delivered in the intervention arm. Primary outcomes were changes in BMI/ weight measured at baseline and at $\geq 6$ months. Data that could be meta-analysed were expressed as mean differences (MD) with 95\% CI otherwise data were reported narratively. Heterogeneity, risk of bias and quality of evidence were assessed.
Results The searches generated 16106 records (duplicates removed). Title and abstract screen excluded 15422 records. 736 records were assessed for eligibility. 105 trials were included: 50 ongoing; 11 awaiting classification, 44 qualitative synthesis and 39 quantitative synthesis. This review included 4682 participants. BMI change at the longest follow-up was MD -1.18 (95\% CI -1.67 to -0.69 ); $\mathrm{p}<0.00001 ; 2774$ participants; 28 trials; low quality evidence. Most studies were multi-disciplinary interventions (BMI MD -1.18 , 95\% CI -1.75 to $-0.61, \mathrm{p}<0.0001 ; 2293$ participants; 22 studies), some studies were diet alone (BMI MD -0.62, 95\% CI -1.29 to $0.06 ; \mathrm{p}=0.07 ; 277$ participants; 3 studies) or exercise alone interventions (BMI MD -1.73 , 95\% CI -3.12 to $-0.34, \mathrm{p}=0.01 ; 229$ participants; 4 studies). Studies undertaken in schools showed a lower effect compered to community and health care settings.

Conclusion Interventions that involve a combination of diet, exercise and behavioural components appear to be an effective treatment option for overweight/obese adolescents. Effects of lifestyle interventions were maintained at 18-24 months follow-up. Results should be interpreted with caution as the evidence was rated as low quality for inconsistency and publication bias.

\section{P93 WHAT ARE THE VIEWS OF OVERWEIGHT AND OBESE ADOLESCENTS (12-17YRS) ATTENDING LIFESTYLE TREATMENT INTERVENTIONS: A QUALITATIVE SYSTEMATIC REVIEW}

HM Jones*, L Al-Khudairy, GJ Melendez-Torres, O Oyebode. Warwick Medical School, University of Warwick, Coventry, UK

\subsection{6/jech-2017-SSMAbstracts. 194}

Background A third of all children in England are overweight or obese. Physical and psychosocial effects of obesity in adulthood are now seen in children and adolescents. Current NICE guidance recommends that obese adolescents attend a familybased multi-component weight management service. Cochrane reviews have shown that lifestyle programmes can be effective. However, weight management interventions recruit $<2 \%$ of the childhood population. For those that do attend weight management interventions, attrition is high. Understanding the views of adolescents is necessary for planning and development of future interventions.

The goals of this systematic review include:

- To explore the views of overweight or obese adolescents aged 12-17 years who have attended a lifestyle treatment intervention.

- To identify what adolescents deem as successful and unsuccessful components of an intervention to inform future practice, as well as research.

Methods This review examined studies of overweight or obese adolescents who have attended a lifestyle treatment intervention. Interventions were single or multi-component and contained one or more diet, physical activity and behavioural elements. Only studies that collected and analysed data qualitatively were included. There were no language restrictions.

Published literature was identified by searching the following databases: MEDLINE, EMBASE, Web of Science, PsycINFO, ASSIA and CINAHL. Reference lists of included studies were screened. 
Titles and abstracts of identified records were assessed independently by two reviewers. Full texts of all potentially relevant papers were retrieved. Two reviewers assessed each full text and reasons for exclusion were recorded. Differences in opinions were resolved by a third reviewer.

Methodological quality was assessed by two reviewers independently as low, medium or high in terms of trustworthiness and reliability of findings. This was done using criteria developed by the EPPI-Centre. Full texts will be opened in NVivo and analysed using thematic synthesis, as described by Thomas and Harden (2008). The certainty of review findings will be assessed using the Confidence in the Evidence from Reviews of Qualitative Research (CERQual) approach.

Results 19230 records were identified for title and abstract screening. 284 full-texts were screened, with 30 included in the final sample.

Conclusion This review is due for completion in June 2017. Findings will inform development of an adolescent weight management intervention in Coventry, West Midlands.

To date, no systematic review has focused on adolescent views of weight management interventions. The views of adolescents should be taken as a starting point when planning future initiatives.

\section{P94 ASSOCIATIONS BETWEEN MATERNAL PREGNANCY, SOCIAL AND LIFESTYLE CHARACTERISTICS AND OFFSPRING BLOOD PRESSURE AT AGE 4/5 IN WHITE BRITISH AND PAKISTANI ORIGIN PARTICIPANTS IN THE BORN IN BRADFORD STUDY}

1J West ${ }^{*},{ }^{1} \mathrm{G}$ Santorelli, ${ }^{1} \mathrm{P}$ Collings, ${ }^{1} \mathrm{~J}$ Wright, ${ }^{2,3} \mathrm{D}$ Lawlor. ${ }^{1}$ Born in Bradford, Bradford Institute for Health Research, Bradford, UK; ${ }^{2}$ School of Social and Community Medicine, University of Bristol, Bristol, UK; ${ }^{3}$ MRC Integrative Epidemiology Unit, University of Bristol, Bristol, UK

\subsection{6/jech-2017-SSMAbstracts. 195}

Background South Asians have an increased risk of stroke and coronary heart disease (CHD) compared to white European populations. High blood pressure (BP) is an important risk factor for CHD and higher diastolic BP has previously been reported in both South Asian adults and children. Our aim was to examine whether maternal pregnancy, social and lifestyle characteristics are associated with BP at age $4 / 5$ and contribute to these differences.

Methods Born in Bradford is a prospective study of children born to 12453 mothers between 2007 and 2010 in Bradford, UK. All mothers completed an oral glucose tolerance test in pregnancy and provided detailed social and lifestyle data. We present data from 2129 White British and 2528 Pakistani mother/offspring pairs from the Born in Bradford study. Associations of BMI, gestational diabetes (GD), fasting and post load glucose, maternal hypertension (HDP), smoking in pregnancy and maternal education with offspring systolic and diastolic BP at age $4 / 5$ were examined. We adjusted our results for sex and age at BP measurement.

Results Pakistani children had lower systolic (mean difference $-0.1695 \% \mathrm{CI}-0.79,0.47$ ) but higher diastolic (mean difference $1.3895 \%$ CI 0.74, 2.03) compared to White British children. In Pakistani children maternal BMI and HDP were strongly associated with higher systolic and diastolic BP but the effect was minimal and weak in White British children. Associations of maternal glucose and BP were consistent with the null hypothesis in both groups. Smoking and education were not associated with BP in either group.

Conclusion Ethnic differences in systolic and diastolic BP are present in children at age $4 / 5$. Consistent with findings in adults, Pakistani origin children have on average lower systolic and higher diastolic BP compared to White British children. Maternal BMI and HDP influence BP in Pakistani children but have minimal effect in White British children. These results suggest a role for some maternal pregnancy and lifestyle exposures in ethnic differences in systolic and diastolic $\mathrm{BP}$ at age $4 / 5$.

\section{P95 ETHNIC VARIATIONS IN CLUSTERING OF ADOLESCENT HEALTH RISK BEHAVIOURS: LATENT CLASS ANALYSIS}

${ }^{1} \mathrm{~A}$ Cassidy ${ }^{*},{ }^{1} \mathrm{O}$ Molaodi, ${ }^{1} \mathrm{M}$ Green, ${ }^{1} \mathrm{~L}$ Moore, ${ }^{2} \mathrm{~S}$ Harding. ${ }^{1} \mathrm{MRC}$ CSO Social and Public Health Sciences Unit, University of Glasgow, Glasgow, UK; ${ }^{2}$ Diabetes and Nutritional Sciences Division, King's College London, London, UK

\subsection{6/jech-2017-SSMAbstracts. 196}

Background There is evidence from existing literature of ethnic variations and clustering in adolescent health risk behaviours. However, it is not known whether there are ethnic variations in health risk behaviour clustering. We aimed to investigate ethnic variations in the clustering of health risk behaviours using the Determinants of young Adults Social wellbeing and Health study (DASH).

Methods Latent class analysis and multinomial logistic regression were used to investigate adolescent health risk behaviour clustering and ethnic variations in clustering in the MRC DASH study. Data were collected from 51 schools across 8 London boroughs. In 2005-06, 4785 pupils were followed up at 14-16 years old. Age, gender, ethnicity, substance use (SU) behaviours (current tobacco and alcohol, and lifetime illicit drug use), daily fruit and vegetables (FV), weekly physical activity (PA), and body mass index (BMI) were recorded.

Results A model with four latent classes was selected (entropy: 0.757). Classes could be characterised as high-SU/high-PA $(\mathrm{n}=232), \quad$ high-SU/low-PA $\quad(\mathrm{n}=811), \quad$ low-SU/PA/high-FV $(\mathrm{n}=1471)$, and low-SU/low-PA/FV $(\mathrm{n}=2260)$.

Using the low-SU/PA/high-FV class as reference males were more likely to be in the high-SU/high-PA class (OR: 1.89; 95\% CI: 1.39-2.57), and less likely to be in the high-SU/lowPA class (OR: 0.56; 95\% CI: 0.47-0.67) than females.

Compared to White UK, Black Caribbean adolescents were less likely to be in the high-SU/high-PA (OR: 0.48; 95\% CI: 0.30-0.76), or high-SU/low-PA (OR: 0.34; 95\% CI: 0.26-0.45) classes. Black Africans were less likely to be in the high-SU/ high-PA (OR: 0.18; 95\% CI: 0.10-0.31), high-SU/low-PA (OR: 0.11 ; 95\% CI: 0.08-0.15), or low-SU/low-PA/FV (OR: 0.55; 95\% CI: 0.44-0.69) classes. Indians were less likely to be in the high-SU/high-PA (OR: 0.36; 95\% CI: 0.19-0.71), or highSU/low-PA (OR: 0.17; 95\% CI: 0.11-0.26) classes. Pakistanis/ Bangladeshis were less likely to be in the high-SU/high-PA (OR: 0.40; 95\% CI: 0.23-0.68), high-SU/low-PA (OR: 0.08; 95\% CI: $0.05-0.13$ ), or low-SU/low-PA/FV (OR: 0.65 ; 95\% CI: 0.50-0.85) classes.

Conclusion Latent class analysis is a valuable method for investigating ethnic variations in adolescent lifestyles. Ethnic minority adolescents tend to be in classes which are characterised by less unhealthy behaviour; patterns also vary between ethnic minority groups. Research needs to investigate risk and protective factors that may explain these ethnic variations to 